\title{
Roles of autophagy in male reproductive development in plants
}

\author{
Shigeru Hanamata ${ }^{1,2+}$, Takamitsu Kurusu ${ }^{1,3,4}{ }^{* \dagger}$ and Kazuyuki Kuchitsu ${ }^{1,4} *$ \\ ${ }^{1}$ Department of Applied Biological Science, Tokyo University of Science, Noda, Japan \\ 2 Department of Integrated Biosciences, University of Tokyo, Kashiwa, Japan \\ ${ }^{3}$ School of Bioscience and Biotechnology, Tokyo University of Technology, Hachioji, Japan \\ ${ }^{4}$ Research Institute for Science and Technology, Tokyo University of Science, Noda, Japan
}

\section{Edited by:}

Diane C. Bassham, lowa State University, USA

Reviewed by:

Byung-Ho Kang, University of Florida, USA

Beatrice Satiat-Jeunemaitre, Centre National de la Recherche Scientifique, France

\section{${ }^{*}$ Correspondence:}

Kazuyuki Kuchitsu, Department of Applied Biological Science, Tokyo University of Science, 2641 Yamazaki, Noda, Chiba 278-8510, Japan e-mail:kuchitsu@rs.noda.tus.ac.jp; Takamitsu Kurusu, School of Bioscience and Biotechnology, Tokyo University of Technology, 1404-1 Katakura, Hachioji, Tokyo 192-0982, Japan

e-mail: kurusutkmt@stf.teu.ac.jp

${ }^{+}$Shigeru Hanamata and Takamitsu Kurusu have contributed equally to this work.
Autophagy, a major catabolic pathway in eukaryotic cells, is essential in development, maintenance of cellular homeostasis, immunity and programmed cell death (PCD) in multicellular organisms. In plant cells, autophagy plays roles in recycling of proteins and metabolites including lipids, and is involved in many physiological processes such as abiotic and biotic stress responses. However, its roles during reproductive development had remained poorly understood. Quantitative live cell imaging techniques for the autophagic flux and genetic studies in several plant species have recently revealed significant roles of autophagy in developmental processes, regulation of PCD and lipid metabolism. We here review the novel roles of autophagic fluxes in plant cells, and discuss their possible significance in PCD and metabolic regulation, with particular focus on male reproductive development during the pollen maturation.

Keywords: autophagic flux, male reproductive development, programmed cell death, tapetum, rice

\section{INTRODUCTION}

Reproductive development, both in animals and plants, is accompanied by drastic changes in metabolism for plentiful supply of nutrients and thus requires its appropriate regulation. In flowering plants, the anther exhibits a four-layered structure composed of the epidermis, endothecium, middle layer, and tapetum. Of these layers, the tapetum provides metabolites and nutrients to pollen grains, microspores, and the pollen coat during their development (Ariizumi and Toriyama, 2011). Tapetum contains triacylglycerol (TAG)-containing lipid bodies, which supply essential lipid components during pollen maturation (Li-Beisson et al., 2010; Murphy, 2012). Recent transcriptomic and bioinformatic analyses have suggested some factors that play roles in regulating lipid metabolism in the anther including a receptorlike kinase, proteases, cell wall-degrading enzymes, cytochrome P450 as well as lipid transfer proteins (Wang etal., 2008; Huang et al., 2009).

Autophagy is an evolutionarily conserved system for degradation and recycling of nutrients (Li and Vierstra, 2012). Intracellular components are enveloped by autophagosomal membranes and fused with the vacuoles/lysosomes, in which they are broken down by lytic enzymes. In animals, this process has recently been suggested to be involved in lipid droplet degradation, and defects in lipid autophagy (lipophagy) have been linked to important metabolic disorders such as fatty liver, obesity, and atherosclerosis (Dong and Czaja, 2011; Liu and Czaja, 2012). In many eukaryotes, autophagy is required for normal development, e.g., for dauer development in nematodes and preimplantation in mice (Tsukamoto et al., 2008; Melendez and Levine, 2009; Mizushima and Komatsu, 2011). In plants, autophagy has been suggested to be involved in seed development and germination, photomorphogenesis, chloroplast maturation, mineral nutrition, hormonal responses, pathogen resistance, stress protection, senescence, and fertile floret development under nutrient-limiting conditions (Ghiglione et al., 2008; Ishida et al., 2008; Chung et al., 2009). However, autophagy-defective Arabidopsis mutants exhibit normal life cycles, and little is known on the roles of autophagy during reproductive development in plants (Yoshimoto, 2012).

Programmed cell death (PCD), a genetically regulated form of cell suicide, plays vital roles in numerous physiological and developmental processes in multicellular organisms (Bozhkov and Lam, 2011; Fuchs and Steller, 2011; Teng et al., 2011). In plants, PCD is essential in various stress responses such as innate immunity against pathogen attack, and development including xylogenesis, pollen maturation, leaf senescence, seed germination, and embryogenesis (Pennell and Lamb, 1997). In angiosperms, cells in numerous reproductive organs undergo PCD during 
reproductive development, e.g., synergids, the anther tapetum, non-functional megaspores, the endosperm, the reproductive primordium, style transmitting tissues, abortive pollens, and antipodal cells (Greenberg, 1996; Pennell and Lamb, 1997; Wei et al., 2002). Homologs of many apoptosis-related genes in animals are not found in plants, and hence, it has been postulated that plants have evolved their own PCD mechanisms (Higaki et al., 2011; Li et al., 2011).

Autophagy is also involved in animal PCD (Shimizu et al., 2004; Tsujimoto and Shimizu, 2005), and has also been suggested to play critical roles in leaf senescence, a developmental PCD specific in plants (Ishida et al., 2008; Van Doorn and Woltering, 2010). Recent studies have shown that tapetal PCD, which plays extremely important roles in fertility, is regulated by a transcriptional network, reactive oxygen species (ROS) as well as activation of proteolytic enzymes in several plant species (Phan etal., 2011; Ma etal., 2012; Niu etal., 2013; Xie etal., 2014).

In this review, we describe temporal changes in autophagic flux that occur in tapetal cells and discuss the relationships between these changes and tapetal degeneration or metabolic regulation, with particular emphasis on plant anther development.

\section{ROLES OF AUTOPHAGY IN RICE ANTHER DEVELOPMENT AND POSSIBLE TAPETAL DEGRADATION DURING POLLEN MATURATION}

The rice autophagy-defective mutants, Osatg7 and Osatg9, show complete sporophytic male sterility and limited anther dehiscence under normal growth conditions, suggesting that autophagy is crucial in reproductive development in rice (Kurusu et al., 2014).
Pollens of the autophagy-defective mutants are premature due to the significant defects in the anther during pollen maturation, while those of the heterozygous plants are normal and mature as the wild type, and the pollination of wild-type stigmas with the heterozygous pollens resulted in normal fertility. These findings indicate that parental tissue or organ defects cause the immature pollen phenotype displayed by the autophagy-defective mutants.

During pollen development, the tapetum provides metabolites and nutrients to pollen grains and microspores. Autophagosomelike structures, including dense globular bodies enclosed within the vacuoles, are detected in the tapetum during the uninucleate stage (Kurusu etal., 2014). On the other hand, the cytoplasm of the autophagy-defective mutants contained basically no autophagosome-like structures, indicating that autophagy is induced at the uninucleate stage in postmeiotic tapetum cells of rice and may be involved in the catabolism of intracellular components such as plastids and lipid bodies during pollen maturation (Kurusu et al., 2014; Figures 1A-D).

As pollens develop, the tapetum is broken down to provide nutrients, metabolites, and sporopollenin precursors to the developing microspores. Defects in tapetal degradation can result in the development of abnormal pollen coats and grains, leading to severe male sterility (Ku et al., 2003; Li et al., 2006; Zhang et al., 2008; Ariizumi and Toriyama, 2011). During tapetal degradation, which is tightly regulated, the characteristic features of PCD such as chromatin condensation, cell shrinkage, endoplasmic reticulum swelling, mitochondrial persistence (Rogers et al., 2005) and nuclear fragmentation (Wang et al., 1999; Vardar and Unal, 2012) are observed.

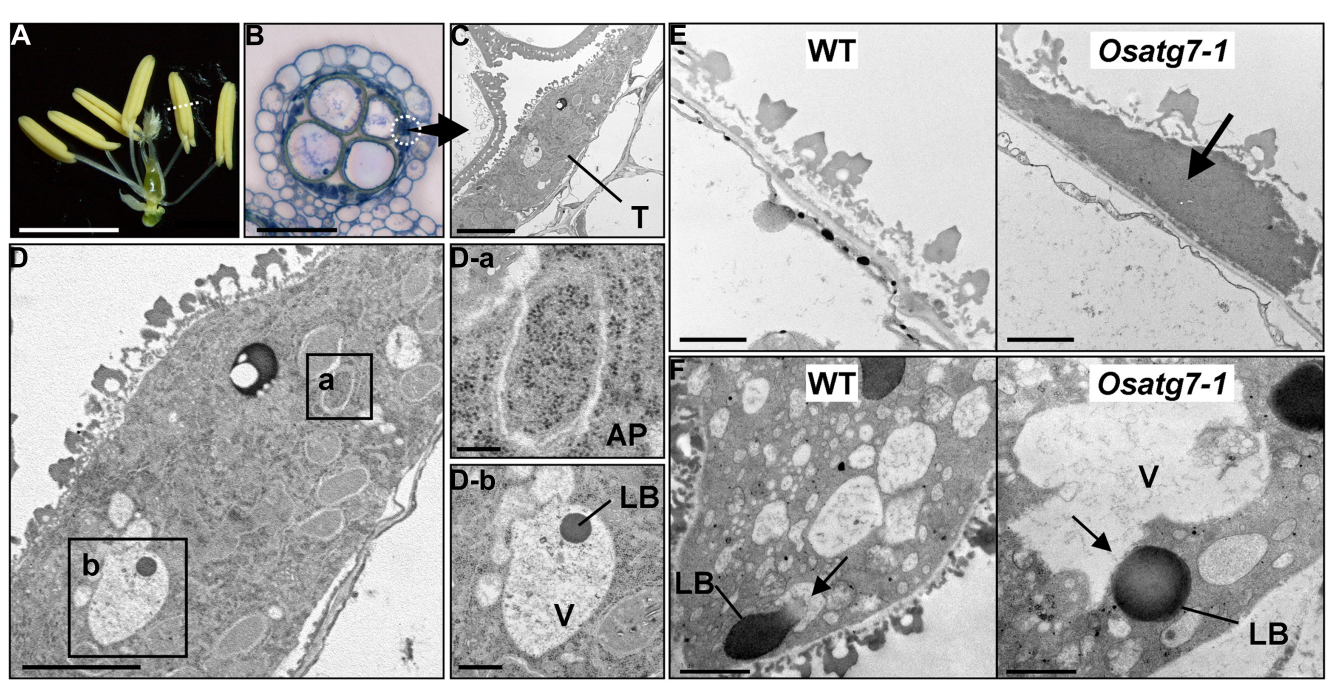

FIGURE 1 | Rice autophagy-defective mutants exhibit sporophytic male sterility, and autophagic degradation within tapetal cells is essential for postmeiotic anther development. (A) Anthers in the wild-type at the uninucleate stage. Scale bar: $3 \mathrm{~mm}$. (B) Transverse sections of wild-type anthers stained with hematoxylin at the uninucleate stage. Scale bar: $50 \mu \mathrm{m}$. (C,D) Autophagosome-like structures and lipid bodies enclosed within the vacuoles detected in postmeiotic tapetum cells during pollen development are depicted. Scale bars: $5 \mu \mathrm{m}$ (C,D) and $1 \mu \mathrm{m}$ (D-a,b).
(E) Potential role of autophagy in tapetal degradation and programmed cell death in rice. Tapetal ultrastructure of the wild-type and the Osatg7-1 mutant at the flowering stage. Scale bar: $1 \mu \mathrm{m}$. Arrows indicate the tapetal cell layers. (F) Lipid bodies directly fuse with the vacuoles at the uninucleate stage in the rice tapetum. Scale bar: $1 \mu \mathrm{m}$. AP, autophagosome; LB, lipid body; $V$, vacuole; $T$, tapetum. Arrows indicate the vacuoles fused with lipid bodies in the wild-type (WT). Similar structure was not observed in the Osatg7-1 mutant. 
Appropriate temporal regulation of tapetal PCD is vital for normal pollen development. The signal initiating tapetal PCD has been suggested to be first produced during the tetrad stage (Kawanabe etal., 2006). Gibberellin controls tapetum degradation (Cheng etal., 2004; Aya etal., 2009). A delay in tapetal breakdown and a switch from PCD to necrosis in the tapeta were observed in an ms1 mutant (Vizcay-Barrena and Wilson, 2006). Pollen wall deposition and the subsequent microspore degeneration failed in a rice mutant in which tapetal degeneration and PCD was retarded (Li et al., 2006, 2011; Zhang et al., 2008).

The rice Osatg7-1 mutant was found to exhibit reduced anther dehiscence, which may contribute to its sterility (Kurusu etal., 2014). Furthermore, the Osatg7-1 mutant exhibits a dense thin layer of tapetal tissue that came into contact with the orbicules, which is not observed in the wild-type even at the flowering stage (Figure 1E), suggesting that whilst the tapetal cell layer of the wildtype is completely degraded, its remnants remain in the mutant (Kurusu et al., 2014). As well as apoptosis, autophagy also plays a role in PCD and cell degeneration in animals (Shimizu et al., 2004; Tsujimoto and Shimizu, 2005).

Autophagic cell death typically involves the formation of double-membrane autophagosomes within the dying cells, which act to remove cell debris (Gump and Thorburn, 2011). Furthermore, a study examining metamorphosis in Drosophila melanogaster found that the destruction of the salivary glands and digestive tract during the latter process were controlled via significant increase in autophagic activity before and during the cell death (Melendez and Neufeld, 2008). Taken together, autophagy may contribute to tapetal breakdown in rice.

Proper timing of tapetal PCD is tightly controlled by an evolutionally conserved transcriptional network mediated by several key transcription factors (e.g., MYB, MADS families) in Arabidopsis and rice. Some proteolytic enzymes including cystein proteases, which play roles in PCD, are often targets of the tapetal transcriptional network (Li et al., 2006; Phan et al., 2011; Niu et al., 2013), suggesting possible involvement of the proteases in the execution of tapetal PCD.

Possible involvement of ROS production has also been suggested to play a role in tapetal PCD. Characteristic ROS accumulation is shown in rice anthers, which was abolished in mads 3 mutant, in which tapetal PCD occurs prematurely (Hu et al., 2011). NADPH oxidase/respiratory burst oxidase homolog (Rboh)-mediated ROS production has recently been suggested to be essential for tapetal PCD progression and pollen development in Arabidopsis (Xie et al., 2014). Expression of Rbohs is also regulated by the transcriptional network regulating the tapetal PCD (Xie et al., 2014). Autophagy-deficient mutants of Arabidopsis such as atg 5 have been shown to over-accumulate ROS in leaves (Yoshimoto et al., 2009). The potential role of autophagy in the regulation of the tapetal transcriptional network as well as ROS signaling is an important topic for future research.

Dynamic reorganization of the vacuoles mediated by actin microfilament has been suggested to play a critical role in executing various PCD in plants (Higaki et al., 2007, 2011). During the tetrad stage, abnormal vacuolization in the tapetum can lead to inappropriate tapetal PCD, resulting in male sterility (Wan et al., 2010).
Tapetal vacuoles have been suggested to provide enzymes capable of degrading the tetrad wall, which are subsequently secreted into the anther locules ( Wu and Yang, 2005). These findings indicate that tapetal vacuoles play significant roles in anther development and PCD. In the tapeta of Lathyrus undulatus L., the vacuolar membrane ruptures and the vacuole collapses at the vacuolated microspore stage, resulting in the release of hydrolytic enzymes and the subsequent destruction of cellular components (Vardar and Unal, 2012). Vacuolar processing enzyme (VPE) is expressed in Arabidopsis anther (Hatsugai et al., 2006), suggesting that VPEmediated proteolysis may be involved in tapetal PCD. Dynamics of the vacuole and autophagy during tapetal PCD should be elucidated in various species in order to understand the molecular mechanisms responsible for autophagy-mediated PCD and its physiological significance in reproductive development in plants.

\section{ROLES OF AUTOPHAGY IN THE REGULATION OF LIPID METABOLISM AND NUTRIENT SUPPLY FROM THE TAPETUM TO DEVELOPING MICROSPORES}

During pollen maturation, lipid bodies containing triacyl glycerols (TAGs) in the tapetum are necessary as a supplier of lipid components (Li-Beisson et al., 2010; Murphy, 2012). However, regulation of lipid metabolism including lipid remodeling remains largely unknown.

Lipophagy; i.e., the autophagic catabolism of lipids, is a selective form of autophagy targeting intracellular lipids. In animals, lipophagy is involved in the breakdown of lipid droplets (Dong and Czaja, 2011), and some important metabolic disorders such as atherosclerosis, fatty liver, and obesity have been found to be associated with defective lipophagy (Dong and Czaja, 2011; Liu and Czaja, 2012).

Lipid bodies enclosed in the vacuoles are detected in rice tapetum cells. Lipid body-like structures in the cytoplasm at the bicellular stage are more abundant in the autophagy-defective mutant than the wild type. A lipidomic analysis of the mutant anthers indicated impaired phosphatidylcholine (PC) editing and lipid desaturation during pollen maturation. These results suggest that in rice anthers, tapetal autophagy is involved in breakdown of lipid bodies and regulation of lipid metabolism, especially Lands cycle-mediated PC editing and desaturation, which affect pollen maturation including pollen coat formation (Kurusu et al., 2014). These findings highlight the significance of autophagy-mediated regulation of lipid metabolism in development. Autophagy is also involved in turnover of peroxisomes (Honig et al., 2012; Shibata et al., 2013; Yoshimoto et al., 2014), which play a role in the regulation lipid metabolism/turnover (Linka and Esser, 2012). The potential role of autophagy in the regulation of lipid metabolism is an important topic for future research.

Since autophagy-defective Arabidopsis mutants complete their own life cycles (Yoshimoto, 2012), autophagy may not play a critical role in the regulation of anther development in Arabidopsis. The most significant difference of the tapetum between dicots and monocots is that dicots have tapetosomes that possess ERderived vesicles and lipid droplets for delivery to the pollen surface (Hsieh and Huang, 2007), while monocots do not form lipidic tapetosomes in tapetal cells. Moreover, many cereals including rice and a vast majority of other plants contain the secretory-type 
tapetum. Their tapetum produces the orbicules termed Ubisch bodies that mainly transport tapetum-derived sporopollenin precursors to developing microspores. However, no such Ubisch bodies have been identified in Brassicaceae including Arabidopsis. A family of lipid transfer proteins is one of the candidates for delivery of sporopollenin precursors from tapetum cells to the developing microspores (Ariizumi and Toriyama, 2011). It may suggest a critical difference in the development of lipidic components in the pollen grains between rice and Arabidopsis. In fact, the structures and components of pollen coat are quite different between cereals and Brassicaceae (Wilson and Zhang, 2009; Ariizumi and Toriyama, 2011). Detailed imaging analyses of various tapetum developmental stages under environmental stress conditions in the Arabidopsis mutants may clarify a novel mechanism for autophagy-mediated PCD and its physiological significance in Arabidopsis.

Several types of autophagy-related pathways have been observed in plant and yeast cells. In macroautophagy and the cytoplasm-to-vacuole transport (CVT) pathway, doublemembrane structure in the cytoplasm fuses with the vacuole. Arabidopsis roots exhibit macroautophagy, in which autophagosomes directly fuse with the vacuoles (Merkulova et al., 2014). On the other hand, in cultured tobacco cells, treatment with E-64c induced the accumulation of autolysosome-like structures around the nucleus, suggesting an alternative autophagic pathway than the macroautophagy (Moriyasu and Ohsumi, 1996). Microautophagy involving invagination of the vacuolar membrane has also been observed (Toyooka and Matsuoka, 2006), however, its dynamics are largely unknown in most plant cells. These results imply that there exist multiple autophagic pathways specific to each cell type, species and tissue in plants.

In rice tapetal cells, lipid bodies directly fuse with the vacuoles at the uninucleate stage, which is distinct from macro- or microautophagy and dependent on OsATG7 (Figure 1F). Further characterization of the dynamics of lipid bodies in the tapetum in various developmental stages in other plant species including Arabidopsis along with genetic analyses may reveal a novel function of autophagy related to lipid metabolism and its physiological significance during postmeiotic anther development.

\section{CONCLUDING REMARKS AND FUTURE PERSPECTIVES}

The GFP-ATG8 fusion protein has been shown as a useful marker to monitor the whole process of autophagy in animals and fungi (Klionsky et al., 2007). A tandem fluorescent protein-tagged ATG8 (RFP-YFP-ATG8)-based non-invasive and semi-quantitative monitoring technique for autophagic flux has recently established in tobacco BY-2 cells (Hanamata et al., 2013). A further advantage of this method is that the autophagic activity of the cells can be quantitatively monitored by simply measuring the fluorescence of cell suspension without using inhibitors such as concanamycin A, a vacuolar $\mathrm{H}^{+}$-ATPase inhibitor. Moreover, turnover and degradation of an autophagy-specific cargo protein cytochrome $b 5$ fused with the photoconvertible fluorescent protein Kikume Green-Red (KikGR) has recently been monitored as a useful marker for autophagic flux in tobacco BY-2 cells (Tasaki et al., 2014). These technical advances in combination with genetic analyses may be useful to characterize environmentally induced autophagy in various specific tissues including tapetum, and reveal novel aspects of autophagy in PCD in plants.

Autophagy is rapidly activated in response to various stimuli as well as developmental processes to induce dynamic reorganization of cytoplasmic components and is involved in the regulation of a wide range of physiological functions in plants. The duration, frequency, amplitude, and selectivity of autophagy seem to affect the specificity of autophagic signaling. Recent studies on ATG proteins have revealed their complex structures, diversity, and regulatory mechanisms, and the identification of ATG8-interacting proteins that recruit specific cargo to the enveloping phagophore sheds new light in the selectivity of autophagy in planta. For future studies, more information on autophagy-mediated signaling events including downstream effectors and signaling cascades is required to better understand their roles. In addition, genetic studies in many plant species and molecular characterization of their associated molecules are necessary to understand their functions.

\section{ACKNOWLEDGMENTS}

This study was supported, in part, by Grants-in-Aid for Scientific Research on Priority Area Nos. 21117516 and 23117718 and Grants-in-Aid for Scientific Research B Nos. 19370023 and 23380027 to Kazuyuki Kuchitsu from MEXT, Japan.

\section{REFERENCES}

Ariizumi, T., and Toriyama, K. (2011). Genetic regulation of sporopollenin synthesis and pollen exine development. Annu. Rev. Plant Biol. 62, 437-460. doi: 10.1146/annurev-arplant-042809-112312

Aya, K., Ueguchi-Tanaka, M., Kondo, M., Hamada, K., Yano, K., Nishimura, M., et al. (2009). Gibberellin modulates anther development in rice via the transcriptional regulation of GAMYB. Plant Cell 21, 1453-1472. doi: 10.1105/tpc.108.062935

Bozhkov, P. V., and Lam, E. (2011). Green death: revealing programmed cell death in plants. Cell Death Differ. 18, 1239-1240. doi: 10.1038/cdd.2011.86

Cheng, H., Qin, L., Lee, S., Fu, X., Richards, D. E., Cao, D., et al. (2004). Gibberellin regulates Arabidopsis floral development via suppression of DELLA protein function. Development 131, 1055-1064. doi: 10.1242/dev.00992

Chung, T., Suttangkakul, A., and Vierstra, R. D. (2009). The ATG autophagic conjugation system in maize: ATG transcripts and abundance of the ATG8-lipid adduct are regulated by development and nutrient availability. Plant Physiol. 149, 220-234. doi: 10.1104/pp.108.126714

Dong, H., and Czaja, M. J. (2011). Regulation of lipid droplets by autophagy. Trends Endocrinol. Metab. 22, 234-240. doi: 10.1016/j.tem.2011.02.003

Fuchs, Y., and Steller, H. (2011). Programmed cell death in animal development and disease. Cell 147, 742-758. doi: 10.1016/j.cell.2011.10.033

Ghiglione, H. O., Gonzalez, F. G., Serrago, R., Maldonado, S. B., Chilcott, C., Cura, J. A., etal. (2008). Autophagy regulated by day length determines the number of fertile florets in wheat. Plant J. 55, 1010-1024. doi: 10.1111/j.1365313X.2008.03570.x

Greenberg, J. T. (1996). Programmed cell death: a way of life for plants. Proc. Natl. Acad. Sci. U.S.A. 93, 12094-12097. doi: 10.1073/pnas.93.22.12094

Gump, J. M., and Thorburn, A. (2011). Autophagy and apoptosis: what is the connection? Trends Cell Biol. 21, 387-392. doi: 10.1016/j.tcb.2011.03.007

Hanamata, S., Kurusu, T., Okada, M., Suda, A., Kawamura, K., Tsukada, E., et al. (2013). In vivo imaging and quantitative monitoring of autophagic flux in tobacco BY-2 cells. Plant Signal. Behav. 8:e22510. doi: 10.4161/psb.22510

Hatsugai, N., Kuroyanagi, M., Nishimura, M., and Hara-Nishimura, I. (2006). A cellular suicide strategy of plants: vacuole-mediated cell death. Apoptosis 11, 905-911. doi: 10.1007/s10495-006-6601-1

Higaki, T., Goh, T., Hayashi, T., Kutsuna, N., Kadota, Y., Hasezawa, S., et al. (2007). Elicitor-induced cytoskeletal rearrangement relates to vacuolar dynamics and 
execution of cell death: in vivo imaging of hypersensitive cell death in tobacco BY-2 cells. Plant Cell Physiol. 48, 1414-1425. doi: 10.1093/pcp/pcm109

Higaki, T., Kurusu, T., Hasezawa, S., and Kuchitsu, K. (2011). Dynamic intracellular reorganization of cytoskeletons and the vacuole in defense responses and hypersensitive cell death in plants. J. Plant Res. 124, 315-324. doi: 10.1007/s10265-011-0408-z

Honig, A., Avin-Wittenberg, T., Ufaz, S., and Galili, G. (2012). A new type of compartment, defined by plant-specific Atg8-interacting proteins, is induced upon exposure of Arabidopsis plants to carbon starvation. Plant Cell 24, 288-303. doi: 10.1105/tpc.111.093112

Hsieh, K., and Huang, A. H. (2007). Tapetosomes in Brassica tapetum accumulate endoplasmic reticulum-derived flavonoids and alkanes for delivery to the pollen surface. Plant Cell 19, 582-596. doi: 10.1105/tpc.106.049049

Hu, L., Liang, W., Yin, C., Cui, X., Zong, J., Wang, X., et al. (2011). Rice MADS3 regulates ROS homeostasis during late anther development. Plant Cell 23, 515 533. doi: 10.1105/tpc.110.074369

Huang, M. D., Wei, F. J., Wu, C. C., Hsing, Y. I., and Huang, A. H. (2009). Analyses of advanced rice anther transcriptomes reveal global tapetum secretory functions and potential proteins for lipid exine formation. Plant Physiol. 149, 694-707. doi: 10.1104/pp.108.131128

Ishida, H., Yoshimoto, K., Izumi, M., Reisen, D., Yano, Y., Makino, A., et al. (2008). Mobilization of rubisco and stroma-localized fluorescent proteins of chloroplasts to the vacuole by an ATG gene-dependent autophagic process. Plant Physiol. 148 , 142-155. doi: 10.1104/pp.108.122770

Kawanabe, T., Ariizumi, T., Kawai-Yamada, M., Uchimiya, H., and Toriyama, K. (2006). Abolition of the tapetum suicide program ruins microsporogenesis. Plant Cell Physiol. 47, 784-787. doi: 10.1093/pcp/pcj039

Klionsky, D. J., Cuervo, A. M., and Seglen, P. O. (2007). Methods for monitoring autophagy from yeast to human. Autophagy 3, 181-206. doi: 10.4161/auto.3678

Ku, S. J., Yoon, H., Suh, H. S., and Chung, Y. Y. (2003). Male-sterility of thermosensitive genic male-sterile rice is associated with premature programmed cell death of the tapetum. Planta 217, 559-565. doi: 10.1007/s00425-003-1030-7

Kurusu, T., Koyano, T., Hanamata, S., Kubo, T., Noguchi, Y., Yagi, C., et al. (2014). OsATG7 is required for autophagy-dependent lipid metabolism in rice postmeiotic anther development. Autophagy 10, 878-888. doi: 10.4161/auto.28279

Li-Beisson, Y., Shorrosh, B., Beisson, F., Andersson, M. X., Arondel, V., Bates, P. D., et al. (2010). Acyl-lipid metabolism. Arabidopsis Book 8:e0133. doi: $10.1199 /$ tab.0133

Li, F., and Vierstra, R. D. (2012). Autophagy: a multifaceted intracellular system for bulk and selective recycling. Trends Plant Sci. 17, 526-537. doi 10.1016/j.tplants.2012.05.006

Li, N., Zhang, D. S., Liu, H. S., Yin, C. S., Li, X. X., Liang, W. Q., et al. (2006). The rice tapetum degeneration retardation gene is required for tapetum degradation and anther development. Plant Cell 18, 2999-3014. doi: 10.1105/tpc.106. 044107

Li, X., Gao, X., Wei, Y., Deng, L., Ouyang, Y., Chen, G., et al. (2011) Rice APOPTOSIS INHIBITOR5 coupled with two DEAD-box adenosine 5'triphosphate-dependent RNA helicases regulates tapetum degeneration. Plant Cell 23, 1416-1434. doi: 10.1105/tpc.110.082636

Linka, N., and Esser, C. (2012). Transport proteins regulate the flux of metabolites and cofactors across the membrane of plant peroxisomes. Front. Plant Sci. 3:3. doi: $10.3389 /$ fpls.2012.00003

Liu, K., and Czaja, M. J. (2012). Regulation of lipid stores and metabolism by lipophagy. Cell Death Differ. 20, 3-11. doi: 10.1038/cdd.2012.63

Ma, X., Feng, B., and Ma, H. (2012). AMS-dependent and independent regulation of anther transcriptome and comparison with those affected by other Arabidopsis anther genes. BMC Plant Biol. 12:23. doi: 10.1186/1471-2229-12-23

Melendez, A., and Levine, B. (2009). "Autophagy in C. elegans," in WormBook, eds J. M. Kramer, D. C. Moerman, and The C. elegans Research Community. Available at: http://www.wormbook.org/

Melendez, A., and Neufeld, T. P. (2008). The cell biology of autophagy in metazoans: a developing story. Development 135, 2347-2360. doi: 10.1242/dev.016105

Merkulova, E. A., Guiboileau, A., Naya, L., Masclaux-Daubresse, C., and Yoshimoto, K. (2014). Assessment and optimization of autophagy monitoring methods in Arabidopsis roots indicate direct fusion of autophagosomes with vacuoles. Plant Cell Physiol. 55, 715-726. doi: 10.1093/pcp/pcu041

Mizushima, N., and Komatsu, M. (2011). Autophagy: renovation of cells and tissues. Cell 147, 728-741. doi: 10.1016/j.cell.2011.10.026
Moriyasu, Y., and Ohsumi, Y. (1996). Autophagy in tobacco suspension-cultured cells in response to sucrose starvation. Plant Physiol. 111, 1233-1241.

Murphy, D. J. (2012). The dynamic roles of intracellular lipid droplets: from archaea to mammals. Protoplasma 249, 541-585. doi: 10.1007/s00709-011-0329-7

Niu, N., Liang, W., Yang, X., Jin, W., Wilson, Z. A., Hu, J., et al. (2013). EAT1 promotes tapetal cell death by regulating aspartic proteases during male reproductive development in rice. Nat. Commun. 4:1445. doi: 10.1038/ncomms2396

Pennell, R. I., and Lamb, C. (1997). Programmed cell death in plants. Plant Cell 9, 1157-1168. doi: 10.1105/tpc.9.7.1157

Phan, H. A., Iacuone, S., Li, S. F., and Parish, R. W. (2011). The MYB80 transcription factor is required for pollen development and the regulation of tapetal programmed cell death in Arabidopsis thaliana. Plant Cell 23, 2209-2224. doi: $10.1105 /$ tpc. 110.082651

Rogers, L. A., Dubos, C., Surman, C., Willment, J., Cullis, I. F., Mansfield, S. D., et al. (2005). Comparison of lignin deposition in three ectopic lignification mutants. New Phytol. 168, 123-140. doi: 10.1111/j.1469-8137.2005.01496.x

Shibata, M., Oikawa, K., Yoshimoto, K., Kondo, M., Mano, S., Yamada, K., et al. (2013). Highly oxidized peroxisomes are selectively degraded via autophagy in Arabidopsis. Plant Cell 25, 4967-4983. doi: 10.1105/tpc.113.116947

Shimizu, S., Kanaseki, T., Mizushima, N., Mizuta, T., Arakawa-Kobayashi, S. Thompson, C. B., et al. (2004). Role of Bcl-2 family proteins in a non-apoptotic programmed cell death dependent on autophagy genes. Nat. Cell Biol. 6, 1221-1228. doi: 10.1038/ncb1192

Tasaki, M., Asatuma, S., and Matsuoka, M. (2014). Monitoring protein turnover during phosphate starvation-dependent autophagic degradation using a photoconvertible fluorescent protein aggregate in tobacco BY-2 cells. Front. Plant Sci. 5:172. doi: 10.3389/fpls.2014.00172

Teng, X., Cheng, W. C., Qi, B., Yu, T. X., Ramachandran, K., Boersma, M. D., et al. (2011). Gene-dependent cell death in yeast. Cell Death Dis. 2:e188. doi: $10.1038 /$ cddis. 2011.72

Toyooka, K., and Matsuoka, K. (2006). "Autophagy and non-classical vacuolar targeting in tobacco BY-2 cells," in Biotechnology in Agriculture and Foresty, Vol. 58, eds T. Nagata, K. Matsuoka, and D. Inze (Berlin: Springer-Verlag), 167-180.

Tsujimoto, Y., and Shimizu, S. (2005). Another way to die: autophagic programmed cell death. Cell Death Differ. 12(Suppl. 2), 1528-1534. doi: 10.1038/sj.cdd.4401777

Tsukamoto, S., Kuma, A., Murakami, M., Kishi, C., Yamamoto, A., and Mizushima, N. (2008). Autophagy is essential for preimplantation development of mouse embryos. Science 321, 117-120. doi: 10.1126/science.1154822

Van Doorn, W. G., and Woltering, E. J. (2010). What about the role of autophagy in PCD? Trends Plant Sci. 15, 361-362. doi: 10.1016/j.tplants.2010.04.009

Vardar, F., and Unal, M. (2012). Ultrastructural aspects and programmed cell death in the tapetal cells of Lathyrus undulatus Boiss. Acta Biol. Hung. 63, 52-66. doi: 10.1556/ABiol.63.2012.1.5

Vizcay-Barrena, G., and Wilson, Z. A. (2006). Altered tapetal PCD and pollen wall development in the Arabidopsis ms1 mutant. J. Exp. Bot. 57, 2709-2717. doi: 10.1093/jxb/erl032

Wan, L., Xia, X., Hong, D., Li, J., and Yang, G. (2010). Abnormal vacuolization of the tapetum during the tetrad stage is associated with male sterility in the recessive genic male sterile Brassica napus L. Line 9012A. J. Plant Biol. 53, 121-133. doi: 10.1007/s12374-009-9095-x

Wang, M., Hoekstra, S., Van Bergen, S., Lamers, G. E., Oppedijk, B. J., Van Der Heijden, M. W., et al. (1999). Apoptosis in developing anthers and the role of $\mathrm{ABA}$ in this process during androgenesis in Hordeum vulgare L. Plant Mol. Biol. 39, 489-501. doi: 10.1023/A:1006198431596

Wang, Y., Wu, H., and Yang, M. (2008). Microscopy and bioinformatic analyses of lipid metabolism implicate a sporophytic signaling network supporting pollen development in Arabidopsis. Mol. Plant 1, 667-674. doi: 10.1093/mp/ $\operatorname{ssn} 027$

Wei, C. X., Lan, S. Y., and Xu, Z. X. (2002). Ultrastructural features of nucleus degradation during programmed cell death of starchy endosperm cells in rice. Acta Bot. Sin. 44, 1396-1402.

Wilson, Z. A., and Zhang, D. B. (2009). From Arabidopsis to rice: pathways in pollen development. J. Exp. Bot. 60, 1479-1492. doi: 10.1093/jxb/erp095

$\mathrm{Wu}, \mathrm{H}$., and Yang, M. (2005). Reduction in vacuolar volume in the tapetal cells coincides with conclusion of the tetrad stage in Arabidopsis thaliana. Sex. Plant Reprod. 18, 173-178. doi: 10.1007/s00497-005-0010-4

Xie, H. T., Wan, Z. Y., Li, S., and Zhang, Y. (2014). Spatiotemporal production of reactive oxygen species by NADPH Oxidase is critical for tapetal programmed 
cell death and pollen development in Arabidopsis. Plant Cell 26, 2007-2023. doi: 10.1105/tpc.114.125427

Yoshimoto, K. (2012). Beginning to understand autophagy, an intracellular self-degradation system in plants. Plant Cell Physiol. 53, 1355-1365. doi: $10.1093 / \mathrm{pcp} / \mathrm{pcs} 099$

Yoshimoto, K., Jikumaru, Y., Kamiya, Y., Kusano, M., Consonni, C., Panstruga, R., et al. (2009). Autophagy negatively regulates cell death by controlling NPR1-dependent salicylic acid signaling during senescence and the innate immune response in Arabidopsis. Plant Cell 21, 2914-2927. doi: 10.1105/tpc.109. 068635

Yoshimoto, K., Shibata, M., Kondo, M., Oikawa, K., Sato, M., Toyooka, K., etal. (2014). Organ-specific quality control of plant peroxisomes is mediated by autophagy. J. Cell Sci. 127, 1161-1168. doi: 10.1242/jcs. 139709

Zhang, D. S., Liang, W. Q., Yuan, Z., Li, N., Shi, J., Wang, J., et al. (2008). Tapetum degeneration retardation is critical for aliphatic metabolism and gene regulation during rice pollen development. Mol. Plant 1, 599-610. doi: 10.1093/mp/ssn028
Conflict of Interest Statement: The authors declare that the research was conducted in the absence of any commercial or financial relationships that could be construed as a potential conflict of interest.

Received: 27 May 2014; accepted: 23 August 2014; published online: 15 September 2014.

Citation: Hanamata S, Kurusu T and Kuchitsu K (2014) Roles of autophagy in male reproductive development in plants. Front. Plant Sci. 5:457. doi: 10.3389/fpls.2014.00457

This article was submitted to Plant Cell Biology, a section of the journal Frontiers in Plant Science.

Copyright (c) 2014 Hanamata, Kurusu and Kuchitsu. This is an open-access article distributed under the terms of the Creative Commons Attribution License (CC BY). The use, distribution or reproduction in other forums is permitted, provided the original author(s) or licensor are credited and that the original publication in this journal is cited, in accordance with accepted academic practice. No use, distribution or reproduction is permitted which does not comply with these terms. 